\title{
Dynamics of $\mathrm{HeHHe}^{+}$Rotational State Changes Induced by Collision with He: A Possible New Path in Early Universe Chemistry
}

\author{
L. González-Sánchez, E. Yurtsever, R. Wester, and F. A. Gianturco*
}

Cite This: J. Phys. Chem. A 2021, 125, 3748-3759

Read Online

ABSTRACT: Ab initio calculations are employed to generate the rigid rotor (RR) potential energy surface (PES) describing the interaction of the linear molecular cation $\mathrm{HeHHe}^{+}$, at its equilibrium geometry, with the neutral $\mathrm{He}$ atom. The resulting interaction is employed to investigate the efficiency of rotational state-changing collisions at the temperatures relevant to the early universe conditions, where the latter molecule has been postulated to exist, albeit not yet observed. The inelastic rate coefficients are found to be fairly large and are compared with those found for another important cation just recently observed in the interstellar medium: the $\mathrm{HeH}^{+}$polar molecule. The possibility for this cation to provide new options to energy dissipation routes under early universe conditions after the recombination era is briefly discussed.

\section{INTRODUCTION}

Chemical species made up of $\mathrm{He}$ and $\mathrm{H}$ atoms are known to be strongly bound when existing as cations so that the possible series of polyatomic structures of the $\mathrm{HeH}_{n}^{+}$are all fairly stably bound and also happen to be composed of the two most abundant elements of our universe. ${ }^{1,2}$ In fact, the strongly bound $\mathrm{HHe}^{+}$cation, which is thought to be the very first diatomic molecule formed when the early universe was cooling down, ${ }^{3,4}$ has been known to exist in laboratories since $1925^{5}$ and has been investigated by high-resolution vibrational ${ }^{6-9}$ and rotational $^{10,11}$ spectroscopy studies quite extensively. Such detailed studies, in fact, have finally enabled the detection of $\mathrm{HHe}^{+}$in interstellar space, an achievement that has been recently reached thanks to the GREAT receiver on-board the SOFIA airplane-borne detector. ${ }^{12,13}$

Several results from mass spectrometric studies ${ }^{14,15}$ and from high-level first-principles quantum-chemical computations ${ }^{1,16-18}$ indicate that the larger $\mathrm{HHe}_{n}{ }^{+}$species (with $n=2-$ 6) are known to be bound and to consist of a relatively strongly bound $\mathrm{HeH}^{+} \mathrm{He}$ core, with a dissociation energy of $D_{0}$ $=3931 \pm 20 \mathrm{~cm}^{-1}$ (refs 2, 16), while additional He atoms are more loosely bound to the central proton by only about 200 $\mathrm{cm}^{-1}$.

The analysis of the properties of the first two terms of the series, $\mathrm{HeH}^{+}$and $\mathrm{HeHHe}^{+}$, is a relevant issue within the context of their possible presence and activity within the interstellar medium (ISM) or within the chemistry of the early universe conditions. The knowledge of their structural properties, as well as their spectroscopic properties, ${ }^{19}$ has therefore been pursued extensively over the years, as discussed before in the literature and will be further illustrated below.
Under the expected conditions of the early universe, which are mostly provided by the results from modeling studies (e.g., see refs 3,13 ), the chemical networks have to rely on the first two elements of the periodic table as the richness of chemistry would only come later when early stars would turn ${ }^{1} \mathrm{H}$ and ${ }^{4} \mathrm{He}$ (along with ${ }^{2} \mathrm{H}$ and ${ }^{3} \mathrm{He}$ ) into carbon, nitrogen, oxygen, and the rest of the broad panoply of species observed today. ${ }^{20,21}$ It is also interesting to note that molecular gases subjected to intense X-rays were found to be profoundly modified by the extensive ionization of its atomic components ${ }^{22}$ so that it stands to reason to expect the presence of highly reactive species like $\mathrm{He}^{+}$and $\mathrm{He}_{2}^{+}$ions and that they in turn could drive primordial chemistry with $\mathrm{H}$ and $\mathrm{H}^{+}$or $\mathrm{H}_{2}$ and $\mathrm{H}_{2}{ }^{+}$.

The structural and spectroscopic properties of $\mathrm{HeHHe}^{+}$ have been recently examined and discussed in the current literature by various authors, ${ }^{23,24}$ where specific experimental identification has been carried out for that same molecule. ${ }^{25}$ We therefore have now a fair amount of information on its likely structure and possible spectroscopic signatures. Furthermore, the formation of $\mathrm{HeHHe}^{+}$has been suggested to take place through reactions of $\mathrm{He}_{2}^{+}$with $\mathrm{H}_{2}$, with the ejection of a hydrogen atom ${ }^{26}$ and the formation of the linear, centrosymmetric $\mathrm{HeHHe}^{+}$molecule in addition to other cations such

Received: February 28, 2021

Revised: April 14, 2021

Published: April 26, 2021 
as $\mathrm{HeH}^{+}$and $\mathrm{HeH}_{2}^{+}{ }^{27}$ Detailed, possible pathways to the creation and destruction in the ISM of this linear cation have been recently analyzed in ref 23 , where different interesting possibilities were put forward as to the formation of this cation.

In the analysis of the primordial chemistry, largely based on numerical models, which are still under refinement or confirmation (e.g., see ref 21), it was suggested that, when the reacting species with neutral $\mathrm{H}$ would be provided as $\mathrm{He}_{2}{ }^{+}$ or $\mathrm{He}^{+}$, the latter ions would be present mainly at high redshifts and at high temperatures since the ensuing expansion and cooling of the primordial gas would efficiently lead to their associative neutralization. On the other hand, a formation channel involving $\mathrm{HeH}^{+}$with $\mathrm{He}$ would be a possible process favored at lower $T$ values, where neutral $\mathrm{He}$ atoms would be present. However, there is an epoch of a few thousand years where $\mathrm{He}$ is present and neutral $\mathrm{H}$ is not. It would therefore be interesting to know if the $\mathrm{HeH}^{+}+\mathrm{He} \rightarrow \mathrm{HeHHe}^{+}$reaction could actually form, in quantity, enough amount of the protonbound complex before the competing reaction: $\mathrm{HeH}^{+}+\mathrm{H} \rightarrow$ $\mathrm{H}_{2}{ }^{+}+\mathrm{He}$ starts taking over and hence favor the destruction of one of the important partners for the $\mathrm{HeHHe}^{+}$-forming chemistry.

Either way, the possible positive outcome which would be of interest for the present study would be that the formation of a very stable, strongly bound linear cation would then have a reasonable chance to survive long enough to contribute to the general dynamical network of molecular energy redistribution and dissipation during the cooling stages of the early universe environment after the recombination era. ${ }^{28}$

In the present work, we therefore intend to investigate as accurately as possible the dynamical option of significant changes of the internal rotational energy content of the title molecule when it interacts with the neutral $\mathrm{He}$ atoms present in the same environment via eq 1

$$
\operatorname{HeHHe}^{+}\left(j=j_{\mathrm{i}}\right)+\mathrm{He}^{\prime} \rightarrow \operatorname{HeHHe}^{+}\left(j=j_{\mathrm{f}}\right)+\mathrm{He}^{\prime}
$$

where the process could lead to either rotational excitation (internal heating) of the cation or its deexcitation (internal cooling) with energy dissipation into the environment. More specifically, we intend to start obtaining from first principles the interaction potential between the ionic molecular partner and the neutral $\mathrm{He}$ atom. The latter potential energy surface (PES) will then be employed to calculate the quantum inelastic cross sections and rate coefficients for the rotational states of the present cation on collision with neutral helium. A comparison will also be shown with the existing data on the behavior of the $\mathrm{HeH}^{+}$cation, the one which has been already detected in the ISM ambient, in collision with both $\mathrm{He}$ and $\mathrm{H}$ atoms. The present new data could thus be employed for upgrading existing kinetic networks with the inclusion of the state-changing rate constants for this new molecular partner within the early universe chemical scenario.

\section{METHODS}

Ab Initio Calculations for the $\mathrm{He} / \mathrm{HeHHe}^{+}$PES. Calculations were carried out using a variety of postHartree-Fock ab initio methods. In one level of analysis, all of the molecular species involved are fully optimized using the coupled-cluster method with full treatment of singles and doubles and an iterative treatment of triples: $\operatorname{CCSD}(\mathrm{T})$ as implemented in the MOLPRO suite of codes. ${ }^{29,30}$ The basis set is chosen to be aug-cc-pVTZ, which was further extended to aVQZ and aV5Z to achieve the complete basis set (CBS) expansion. The harmonic zero-point-energy (ZPE) corrections were included in all of these calculations.

The structural parameters of the molecular partner produced by our calculations yielded a $\mathrm{He}-\mathrm{H}$ bond distance of $0.9249 \AA$ and therefore an overall $\mathrm{He}-\mathrm{He}$ distance of $1.8498 \AA$. These values are in good accordance with those reported in ref 23. The two-dimensional (2D) potential energy surface was sampled by mapping the $(R, \theta)$ space over 10 evenly spaced angles from 0 to $90^{\circ}$ and over radial distances from 2.05 to $20.00 \AA$, for a total of 750 points. The angle $\theta$ is between the molecular bond and the radial distance from the center of mass of the cation (i.e., the $\mathrm{H}$ atom) and the impinging $\mathrm{He}$ atom. The full PES was then obtained by duplication of the points from 90 to $180^{\circ}$, thereby mapping it with a total of about 1500 points. When the angular sectors for additional angles, beyond the initial set mentioned earlier, were added to test the calculations, we found that the ensuing scattering expansion (see below) did not change significantly.

We report in Figure 1 a 3D representation of our computed interaction, which provides a pictorial and clear view of the

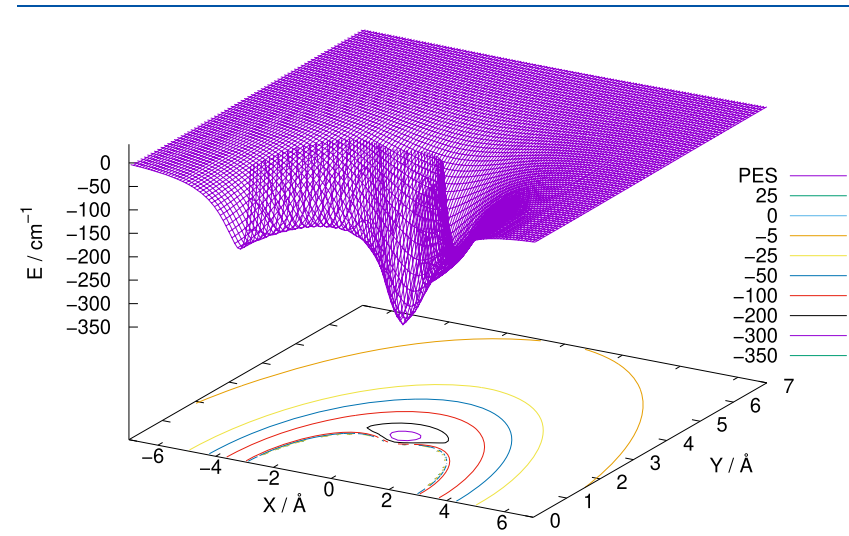

Figure 1. 3D presentation of the PES for the title molecular cation and the neutral He atom. The origin of the coordinates is taken at the center of mass of the molecular ion.

marked anisotropy of the present PES, a feature of the interaction which will be important when discussing the quantum dynamics of the rotational energy transfer collisions. We also see that the most stable structure of the complex corresponds to the $T$-shaped geometry with the incoming neutral $\mathrm{He}$ pointing at the $\mathrm{H}$ atom at the center of mass of the partner cation, as expected from simple electrostatics, given the location of the positive charge in the cation. The present value found for that well depth is about $-350.0 \mathrm{~cm}^{-1}$ at a distance of about $1.137 \AA$ A.

To gain additional information on the scattering-oriented features of the present PES, we have also expanded the potential in terms of Legendre polynomials

$$
V(R, \theta)=\sum_{\lambda} V_{\lambda}(R) P_{\lambda}(\cos \theta)
$$

The resulting expansion coefficients $\left(V_{\lambda}\right.$ 's) provide radial functions, which are directly linked, in the following section, to the angular dynamical torque generated by the present PES during the collisions, the one causing changes of the initial rotational states of the cation. Some of these radial coefficients are plotted in Figure 2, which shows the relative strength of each of them over the expected radial range of action of the 


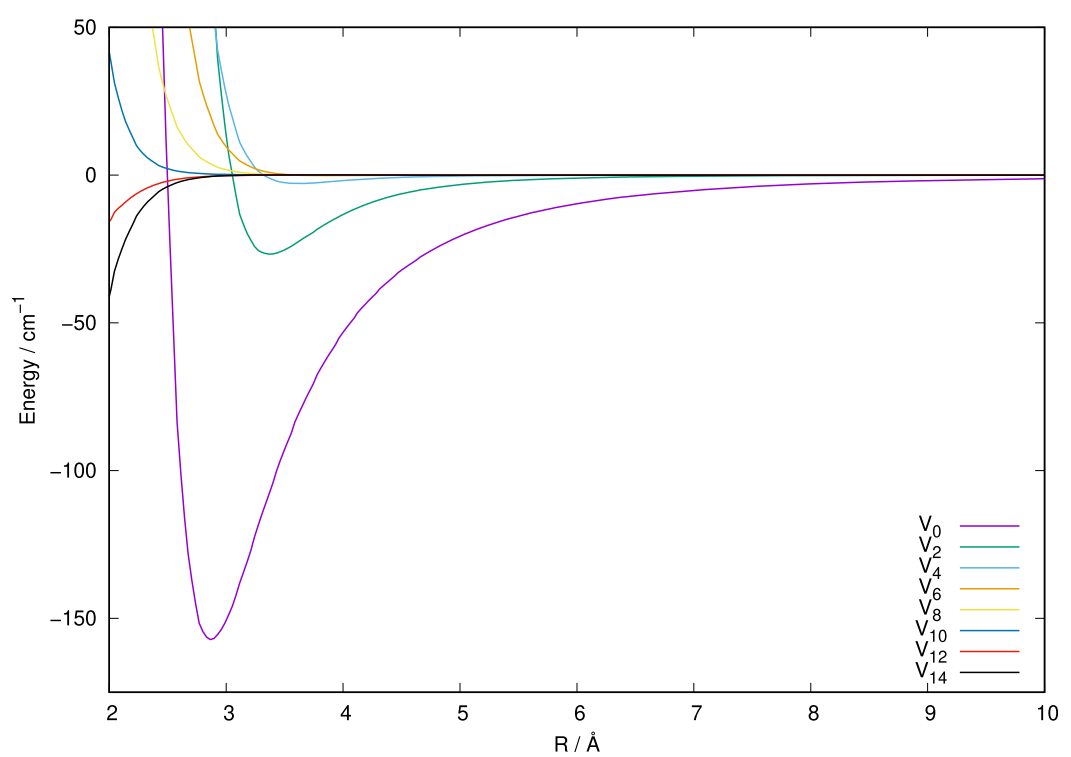

Figure 2. Multipolar expansion coefficients for the RR-PES potential computed in the present study.

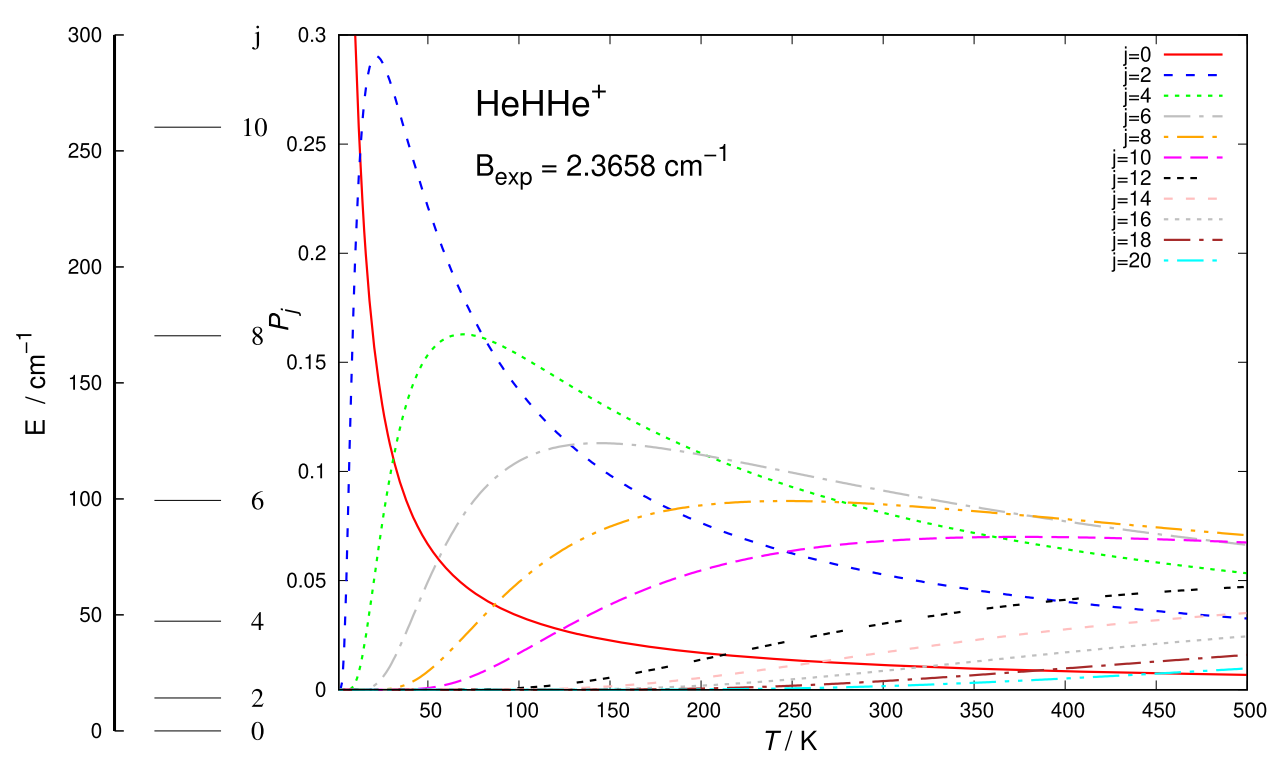

Figure 3. Boltzmann-averaged distributions of the rotational states of the $\mathrm{HeHHe}^{+}$cation as a function of temperature. The rotational constant employed for the averaging is reported.

potential. The dominant attractive features shown by the $\lambda=0$ isotropic coefficient reflect one important aspect of the physics of the interaction of the cation, which involves the spherical polarizability of the $\mathrm{He}$ partner, leading to the possible formation of bound complexes. We shall not be discussing here the mechanism of formation of such complexes as they will be the subject of a different publication dealing with the formation mechanisms of the title cation.

On the other hand, the significant attractive wells shown by the radial $\lambda=2$ and 4 anisotropic coefficients indicate that rotational transitions involving $\Delta j=2$ and 4 will be more efficiently activated during collisions since their angular coefficients would directly couple the $j$ and $j \pm 2,4$ rotational levels. Hence, the data in Figure 2 allow us to explicitly judge the relative strength of the multipolar coefficients of the $2 \mathrm{D}$ PES in dynamically directing flux into the different inelastic final channels. The actual numerical raw grid of the individual radial coefficients has been interpolated to obtain a polynomial representation that was further expanded asymptotically, within the employed scattering code, to correctly represent the spherical and dipolar leading terms of the long-range interaction. An additional exponential extrapolation was employed to reach shorter distances in the repulsive region.

It is also of interest to look at the properties of the $\mathrm{HeHHe}^{+}$ molecular ion in terms of the energy spacings between its lower rotational levels and the Boltzmann-averaged distributions of their relative populations over the significant range of temperatures for the present processes. The results are reported in Figure 3, where one sees how many of the rotational states can be populated under equilibrium conditions up to $500 \mathrm{~K}$. Note that the symmetry of the present rotor only allows for even values of the rotational quantum number $j$. This range of $T$ values will be the actual range of interest for the low redshift regimes, where neutral $\mathrm{He}$ is expected to be more present. ${ }^{21}$ These features will also play a role when discussing, in the following section, the results from 

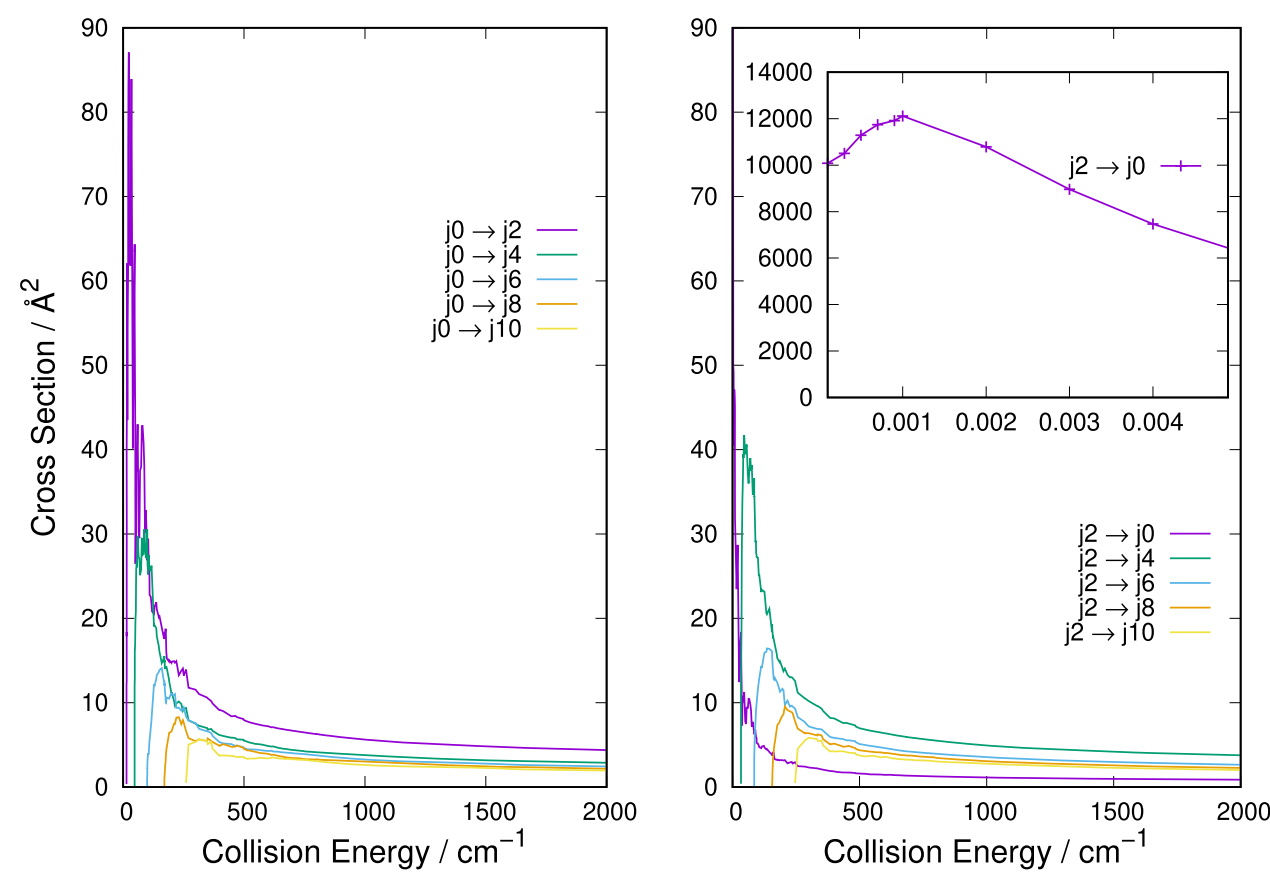

Figure 4. State-to-state computed partial integral cross sections for the inelastic processes that start from the $j=0$ rotational state (left) and for those processes that originate from the $j=2$ state (right). The inset in the right panel shows an enlarged view of the $\Delta j=-2$ deexcitation cross section between the two lowest levels near the threshold energy.

the quantum dynamics and the relative behavior of the different state-changing collisional rate constants between the rotational states of the title cation.

The following section shall report a short outline of the quantum method we have employed to calculate inelastic cross sections and rate coefficients for the present system.

Quantum Dynamics for Rotational State-Changing Collisions. The standard time-independent formulation of the coupled-channel (CC) approach to quantum molecular scattering will not be repeated here in detail (see, for example, ref 31 for a general formulation) since the general literature on the actual computational methods has been very extensive. For a selected set of references over the more recent years, see, for instance, refs 32-36. Since we have already discussed the method we employ in many of our earlier publications, ${ }^{37-39}$ only a short outline will be given below.

For processes where no important chemical modifications would occur in the molecule by the interaction with this impinging projectile at the energies of interest, the total scattering wave function can be expanded in terms of asymptotic target rotational eigenfunctions (within the rigid rotor approximation), which are taken to be spherical harmonics and whose eigenvalues are given by $B j(j+1)$, where $B$ is the rotational constant for the closed-shell $\mathrm{HeHHe}^{+}$ anion: $2.3658 \mathrm{~cm}^{-1}$ as given in ref 23. The channel components for the CC equations are therefore expanded into products of total angular momentum $J$ eigenfunctions and of radial functions to be determined via the solutions of the CC equations, ${ }^{37,38}$ i.e., the familiar set of coupled, secondorder homogeneous differential equations

$$
\left(\frac{\mathrm{d}^{2}}{\mathrm{~d} R^{2}}+\mathbf{K}^{2}-\mathbf{V}-\frac{\mathbf{l}^{2}}{R^{2}}\right) \boldsymbol{\psi}^{J}=0
$$

In the above equation, the matrices $\mathbf{K}^{2}, \mathbf{V}$, and $\mathbf{l}^{2}$, respectively, represent the kinetic energy channel values, the coupling potential terms, and the centrifugal angular momenta values for each of the coupled channels in the equations.

A variety of scattering observables are therefore obtained in the asymptotic region of the interaction between partners, where the log-derivative matrix has a known form in terms of free-particle solutions and unknown mixing coefficients. ${ }^{31}$ Therefore, at the end of the propagation of the radial solutions from the coupled equations, one can use the log-derivative matrix to obtain the K-matrix by solving the following linear system

$$
\left(\mathbf{N}^{\prime}-\mathbf{Y N}\right)=\mathbf{J}^{\prime}-\mathbf{Y J}
$$

where $\mathbf{J}(R)$ and $\mathbf{N}(R)$ are the matrices of Riccati-Bessel and Riccati-Neumann functions. ${ }^{38}$

From the K-matrix, the S-matrix is easily obtained, from which one can extract the individual state-to-state cross sections. We have already published an algorithm that modifies the variable-phase approach to solve that problem, specifically addressing the latter point, and we refer the interested reader to those references for further details. ${ }^{37,38}$

In the present calculations, we have generated a broad range of state-to-state rotationally inelastic cross sections from the interaction of $\mathrm{HeHHe}^{+}$with $\mathrm{He}$. We shall also be comparing the new results with our earlier ones on the $\mathrm{HeH}^{+}$cation with $\mathrm{He}$ atoms ${ }^{40}$ to provide a quantitative assessment with a cation already observed in the ISM environment.

Specifically, our present calculations have used up to 26 terms in the multipolar expansion of the initial PES to account for the strong anisotropy of the present system. The radial range of integration has been varied depending on the collision energy considered and has extended from 1.5 to $100 \AA$, and the number of coupled channels in the coupled equations went up to 22 rotational states. In the global partial wave expansions of the calculations, the range of $J$ values from $J=0$ up to $J_{\max }=$ 100 was found to be sufficient to reach convergence of the numerical values of the state-to-state opacity functions. The 

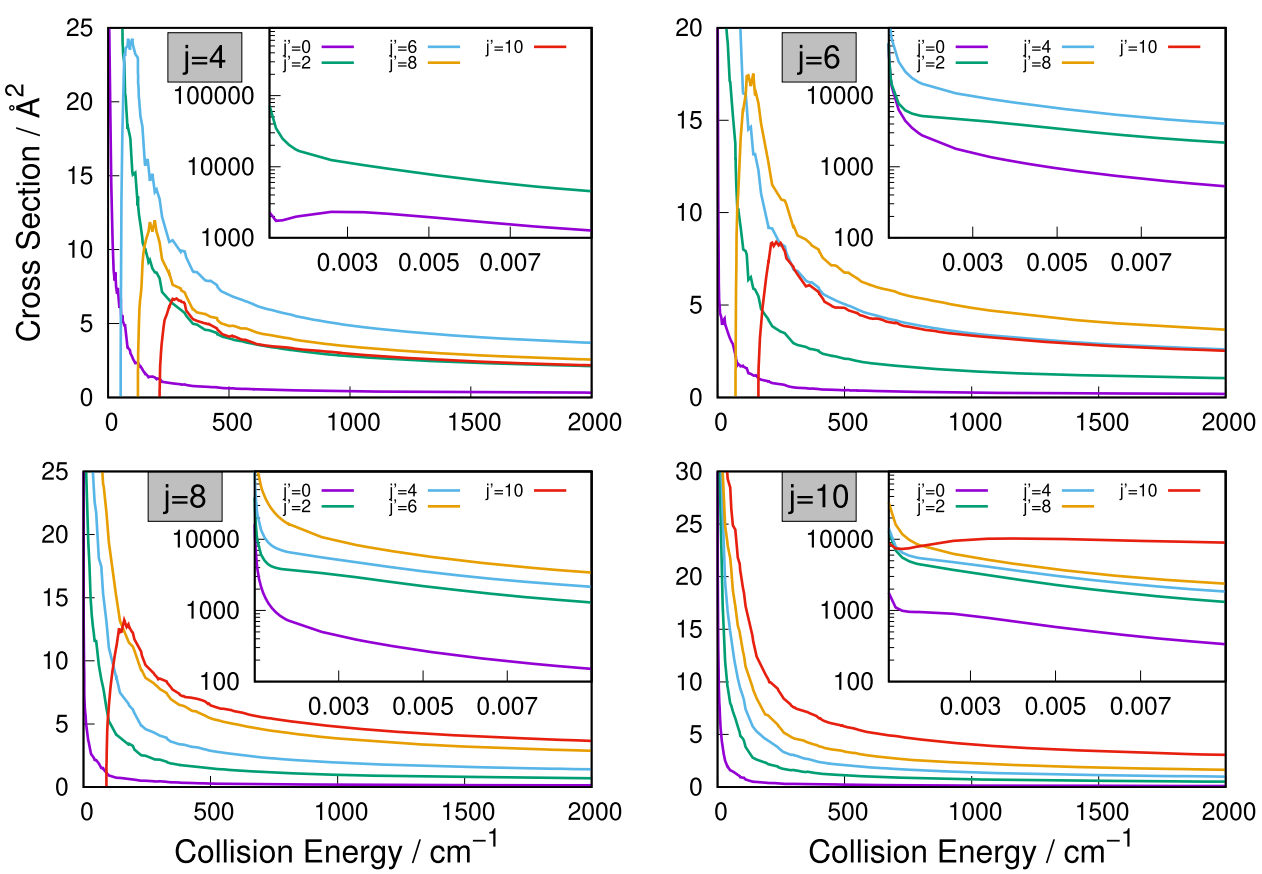

Figure 5. State-to-state computed partial integral cross sections for the excitation and deexcitation processes. The four panels indicate statechanging processes that originate from different initial rotational states of the cation. The top left and top right panels show transitions from the initial $j=4$ and $j=6$ states, respectively, while the bottom left and bottom right panels present transitions from the $j=8$ and $j=10$ rotational states, respectively. The insets in all panels show the cross section behavior near the threshold energies.

range of energies considered for the cross sections has increased from 0.0001 up to $2260.00 \mathrm{~cm}^{-1}$. Transitions have been calculated between $j=0$ and $j=10$ with all of the intermediate values taken into account for producing final inelastic cross sections.

Once the state-to-state inelastic integral cross sections are known, the rotationally inelastic rate constants $k_{j \rightarrow j^{\prime}}(T)$ can be evaluated as the convolution of the cross sections over a Boltzmann distribution of the relative collision energies

$$
k_{j \rightarrow j^{\prime}}(T)=\left(\frac{8}{\pi \mu k_{\mathrm{b}}^{3} T^{3}}\right)^{1 / 2} \int_{0}^{\infty} E \sigma_{j \rightarrow j^{\prime}}(E) \mathrm{e}^{-E / k_{\mathrm{B}} T} \mathrm{~d} E
$$

where $\mu$ is the reduced mass of the system, given by 2.77166 $\mathrm{amu}$, and $k_{\mathrm{B}}$ is the usual Boltzmann constant. The numerical quadrature was carefully controlled over the required range of computed cross sections, which we shall be reporting in the next section, using numerical polynomial interpolation/ extrapolation to reach the necessary cross section threshold values and the values at the highest energies included.

The interplay between the effect of the reduced mass value, appearing in the denominator in the equation above, and the structural strengths of the corresponding PES multipolar coefficients given in Figure 2, has an important effect on the inelastic dynamics since they work in opposite directions in producing the final values of the inelastic rate constants. We shall see from our results that the marked anisotropy of the interaction potential is the property that is mainly driving the large state-changing probabilities we have found for this molecule.

\section{RESULTS AND DISCUSSION}

The quantum calculations of the relevant rotationally inelastic cross sections of the present cation were carried out over a broad range of energies expected to be sufficient to describe the lower temperature and lower redshift values at which the existence of the title molecule could occur under early universe conditions.

Inelastic Cross Sections and Rate Coefficients. More specifically, the data shown in Figure 4 report the excitation processes with increasing $\Delta j$ values, keeping in mind that the symmetry of the present rotor only allows for even values of the rotational quantum numbers, as discussed before. We show there as examples the state-changing processes originating from the $j=0$ and $j=2$ states.

When looking at the excitation cross sections from the lowest rotational state of this cation, we notice that, as discussed earlier, the process involving the direct $\Delta j=2$ transition, i.e., the one chiefly activated by the direct dynamical coupling via the multipolar coefficient with $\lambda=2$, is by far the largest inelastic cross section at threshold and remains so up to the highest collision energy we have considered in this study. In that same threshold energy region, we see that the excitation process largely controlled by the direct multipolar coefficient with $\lambda=4$, the one previously seen to be the next more important coupling torque for the present system, is very large and comparable in size to the process discussed before. As the collision energy increases, the latter inelastic cross section with $\Delta j=4$ becomes smaller than the former one (with $\Delta j=2$ ) but shows a very similar energy dependence. It is also interesting to note in that panel that all of the other inelastic processes, involving increasingly larger changes of the $\Delta j$ values up to $\Delta j$ $=8$, become increasingly smaller near the threshold region, but at higher energies, they remain comparable in size with the state-changing processes associated with the smallest $\Delta j$ values. This is an interesting result which suggests that the efficiency of energy uptake via collisions by the present cation could involve a larger range of possible transitions than would be expected from what other ionic systems have shown in our earlier calculations. ${ }^{40}$ 


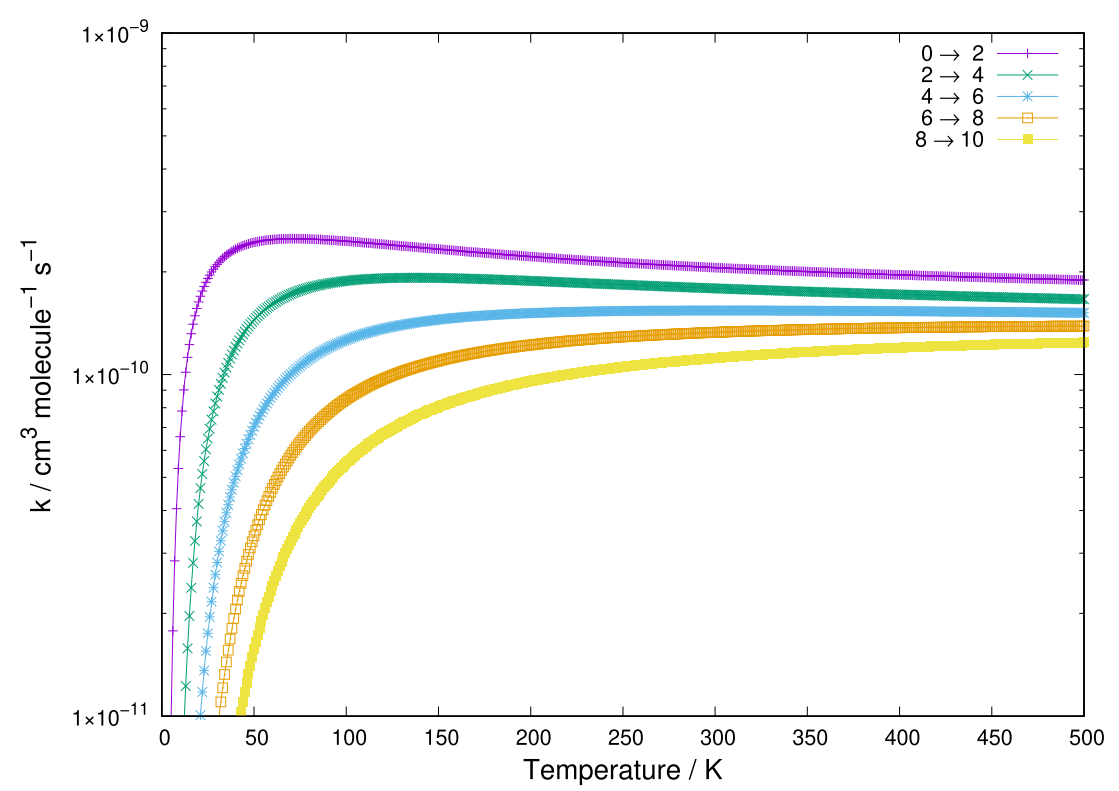

Figure 6. Computed rotationally inelastic excitation rate coefficients associated with the $\Delta j=2$ transitions starting from the $j=0$ level to the $j=8$ level.

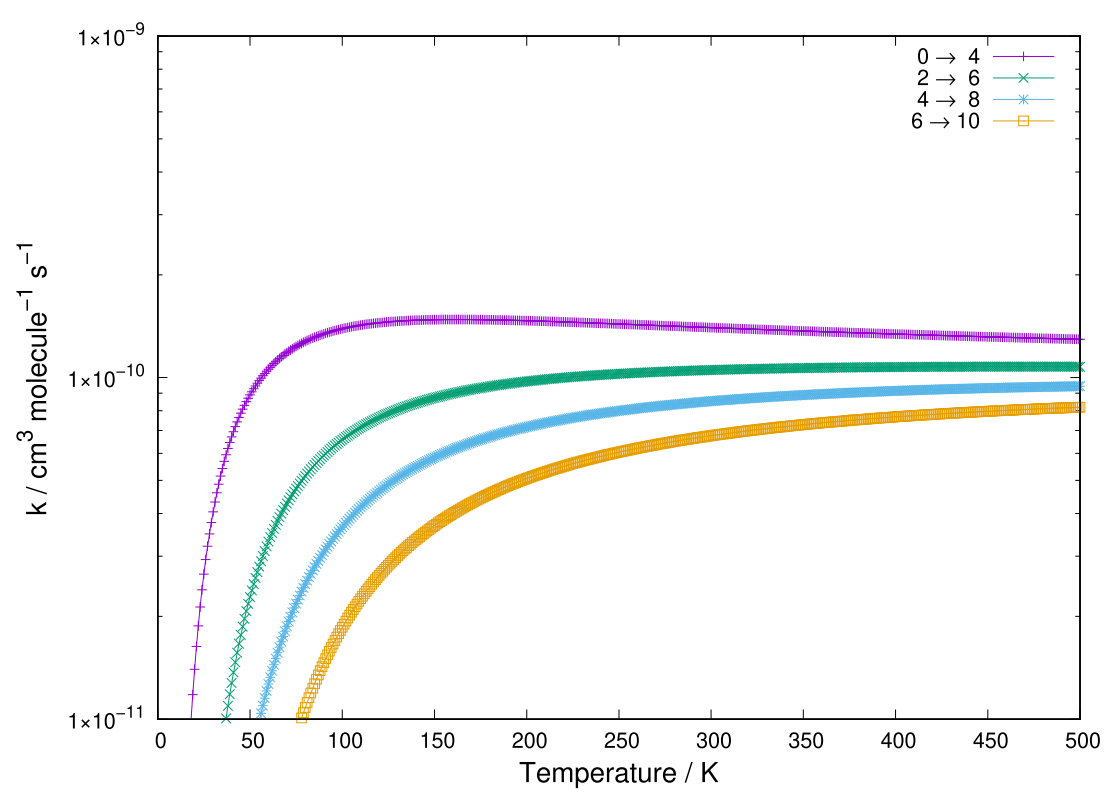

Figure 7. Same computed quantities as in Figure 6, but here showing excitation rate coefficients for the $\Delta j=4$ transitions, starting from the $j=0$ level to the $j=8$ level.

The results shown in Figure 4 (right) report a sequence of state-changing processes with transitions from the initial $j=2$ rotational state of the cation. The specific threshold behavior shown in the inset also indicates that the deexcitation process associated with the $\Delta j=-2$ transition is the largest of all processes, remaining more than 2 orders of magnitude larger than all of the others at collision energies within fractions of $\mathrm{cm}^{-1}$. The smallest energy gap involved, more significant at the lowest collision energies where the corresponding cross section is by far the largest, is therefore causing larger cross sections. However, as the collision energy moves above the various threshold energies, all of the inelastic cross sections become comparable in size, and the deexcitation process increasingly becomes the smallest cross section in comparison to the excitation transitions from the same $j=2$ state. This behavior indicates that the increases in the energy spacing between the higher rotor states reduce the interaction time between partners when deexcitation is considered while they increase it for excitation processes. Hence, the adiabatic criterion qualitatively suggests that energy transfer efficiency is best when the forces induced by the collision are on the same time frame as that of the internal motion. Thus, excitation and deexcitation processes change their relative importance depending on the considered range of collision energies in relation to the energy gaps involved (e.g., see ref 41). This behavior was also found in our earlier work with different cations $^{42}$ and will be discussed when analyzing the inelastic rate coefficients.

Additional calculations are reported in Figure 5, where we show a more extended range of collision energies for which the 


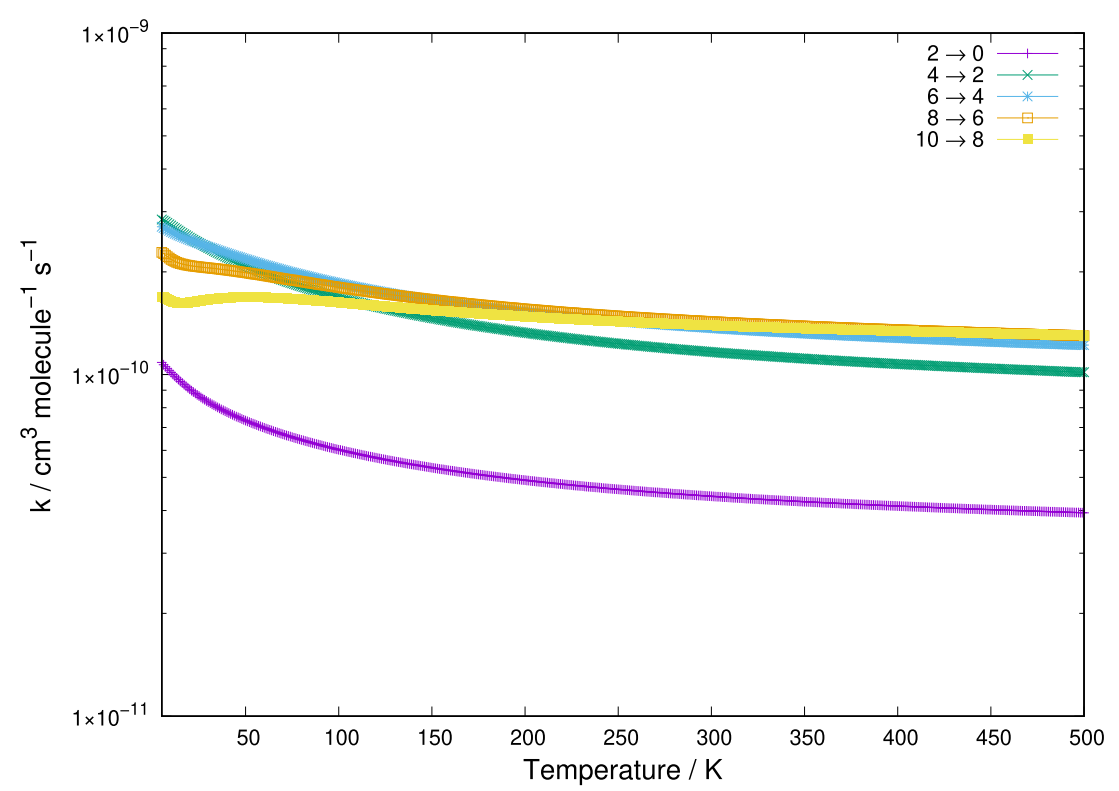

Figure 8. Same computed quantities as in Figure 6, but here showing deexcitation rate coefficients for the $\Delta j=-2$ transitions, starting from the $j=$ 2 level to the $j=10$ level.

state-changing processes have been investigated. The insets in each of the panels indicate the large cross section values found near the corresponding threshold for the deexcitation processes. The initial rotational states considered for each set of calculations increase along the panels, from the top left to the bottom right so that each panel shows both excitation processes and deexcitation processes occurring during collisions from that particular initial state. Such choices help reveal the differences in energy-storing and energy-releasing collisional events when they involve the network of rotational states of this cation. We have seen from the Boltzmann-type populations presented in Figure 3 that the lower rotational states up to $j=10$ should be significantly populated at the temperature of interest. Although we do not know as yet whether to expect the present molecule to be within local thermal equilibrium (LTE) for rotational states, we shall take it as a qualitative initial indicator for helping us in choosing the most important rotational states to be considered here.

Figure 5 shows that at the thresholds for the opening of the excitation (energy-storing) processes, their cross sections are dramatically larger than those pertaining to the deexcitation (energy-releasing) processes. When the collision energy moves up from a few $\mathrm{cm}^{-1}$ to the energies above the inelastic excitation thresholds, and then up to about $2000 \mathrm{~cm}^{-1}$, we clearly see that the excitation cross sections remain dominant in size while the deexcitation channels decrease as the energy increases. Such behavior is again in line with the adiabatic criterion mentioned for the previous figure and concerning the interplay between interaction times and involved energy gaps as already discussed in ref 41 .

As mentioned in the previous section, the inelastic cross sections are the starting ingredient for the production of the rate coefficients associated with those state-changing processes over the range of temperatures of interest for kinetic modeling studies. The present calculations were carried out for the rotational states discussed in the previous section, and the resulting rate constants are given in Figures 6-8. Because of the specific energy-dependence behavior we found for the inelastic cross sections, the actual numerical quadrature to obtain the corresponding rate coefficients from the lowest temperatures and up to $500 \mathrm{~K}$ was very carefully controlled to make sure that all values, at all temperatures, were numerically converged within a few percents and that the final rate values were stabilized.

The data reported in Figure 6 clearly show that the relative size of the computed rate coefficients decreases with the increase of the energy gaps between different transitions. The largest transition energy, associated with the $j=8$ to $j=10$ rotational excitation, is nearly 1 order of magnitude larger than the one for the $j=0$ to $j=2$ rotational excitation. Accordingly, the latter rate constant is on average about twice as large as the former. This inverse relation between size and energy gap uniformly applies to all of the rate coefficients shown in the figure. Furthermore, the marked anisotropy discussed earlier for the employed PES is also causing here that the range of values of all rate coefficients remains fairly large at the temperatures considered, once beyond their relative threshold values, so that the size differences just mentioned are fairly small in relative terms.

We have mentioned earlier that the direct coupling potential for the $\Delta j=4$ transitions is close in strength and radial range to the one that applies the direct rotational torque for the $\Delta j=2$ transitions. As a result of this feature, we therefore see that the excitation rate constants reported in Figure 7 and showing the excitation processes for the $\Delta j=4$ state-changing collisions are not significantly smaller than those for the $\Delta j=2$ excitations. At the highest temperatures shown in Figures 6 and 7, we see that the values in Figure 7 are only a few percents smaller than those presented in Figure 6. We can therefore argue that collisional excitation processes for the title cation in collision with $\mathrm{He}$ atoms would efficiently cover a fairly broad range of internal state excitations, thereby contributing to the internal energy-storing step under expected conditions at the time of the recombination era.

Another set of useful comparisons can be seen from the sampling of deexcitation rate coefficients reported in Figure 8.

The deexcitation rate coefficients reported in Figure 8 clearly show that the rate values increase as the initial rotational state 


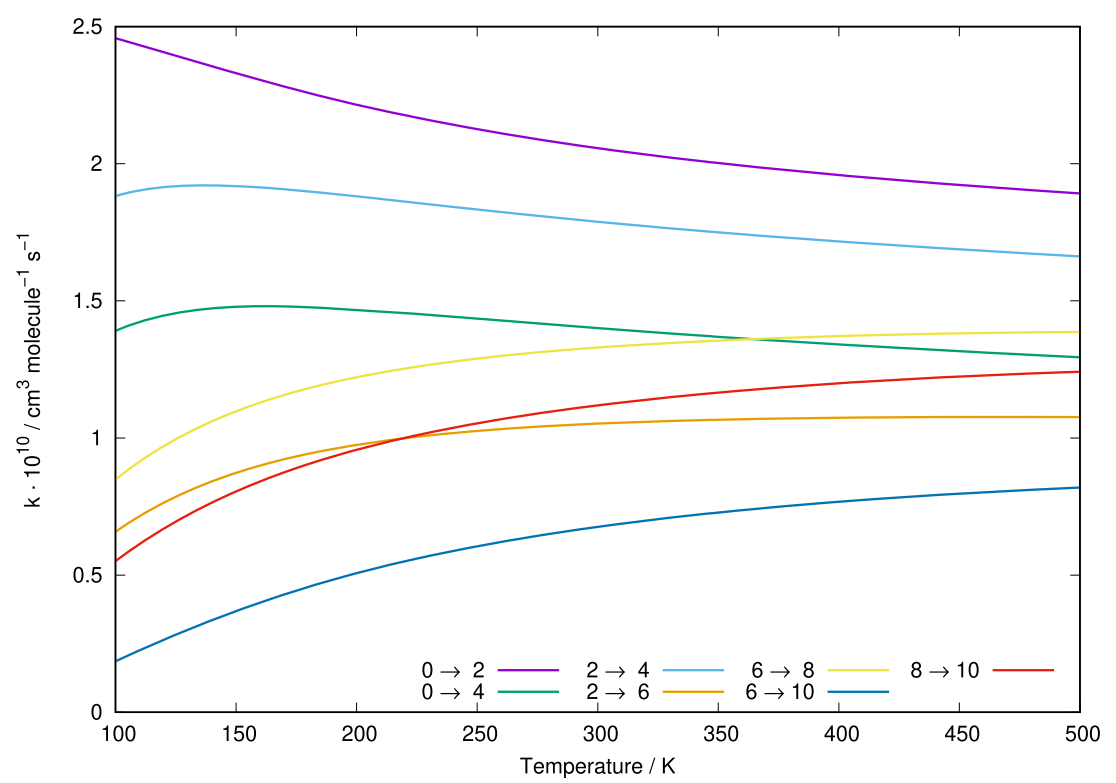

Figure 9. Enlarged view of the computed excitation rate constants from different initial levels covering the range examined in this work and involving both $\Delta j=2$ and $\Delta j=4$ transitions.

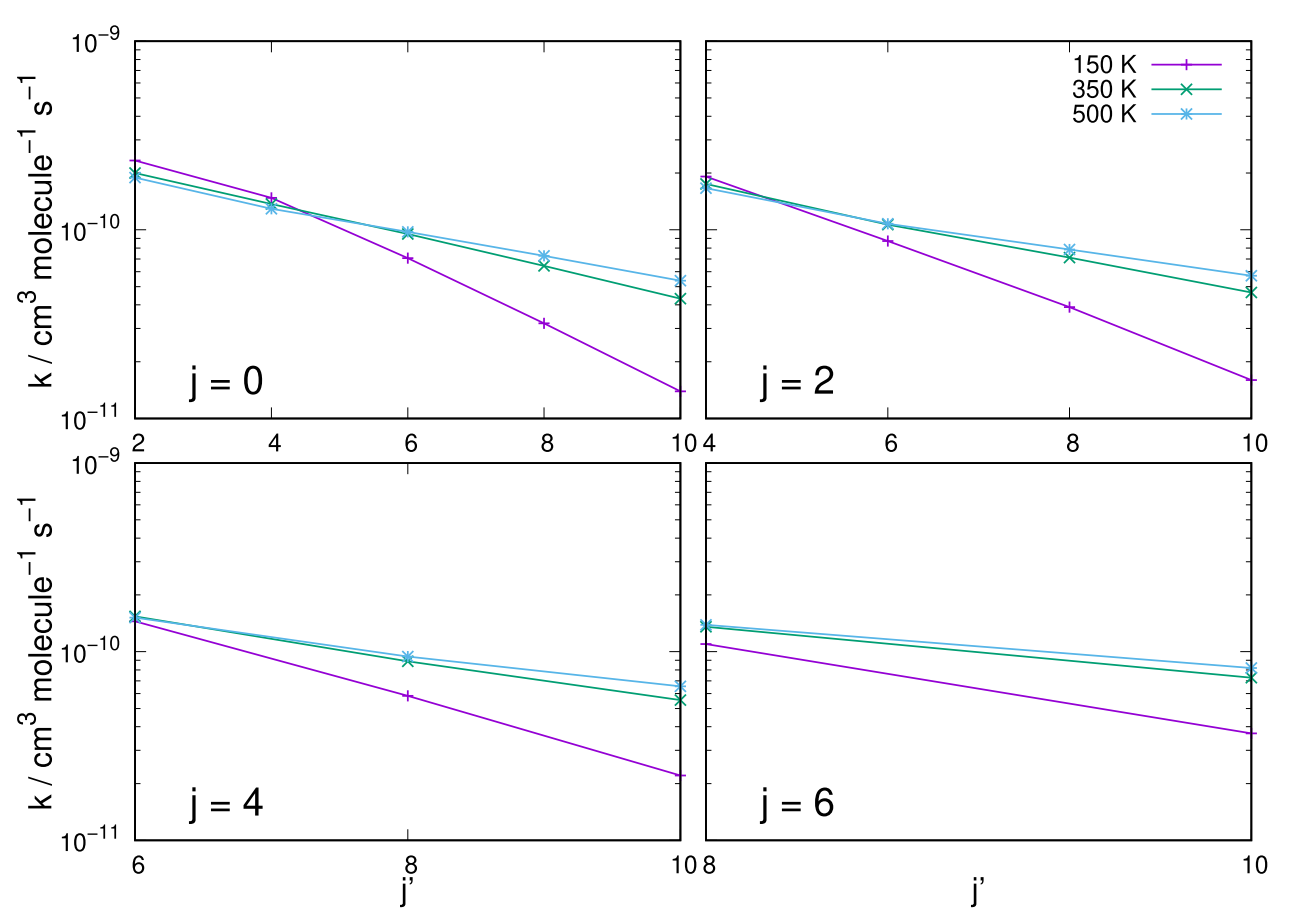

Figure 10. Behavior of the computed excitation rate constants at three different temperatures and for transitions that start from different initial rotational states. Each panel reports all three different temperatures for a given initial state of the cation.

of the cation increases, with the deexcitation process from the lowest initial state possible $(j=2)$ being that with the smallest rate coefficient. These findings are in the opposite order with respect to the excitation processes discussed in Figures 6 and 7, where the excitation rates from the lower levels were the larger ones. This feature has to do with the opposite role of the energy gap value, in either excitation transitions or deexcitation transitions, with respect to the dynamics being more or less impulsive after collision depending on the value of the residual/released relative kinetic energy. Thus, the deexcitation processes remain efficient and are larger when more internal energy is being released into the relative kinetic energies of the partners (see again ref 41 for an earlier discussion of this point).

Further Indicators from Inelastic Rate Coefficients. To provide another comparative view on the behavior of the collisional rotational state-changing dynamics presented in previous figures, we report in Figure 9 an enlarged comparison of a subset of excitation rate constants from different initial rotational states of the cation, undergoing both $\Delta j=2$ and $\Delta j$ $=4$ excitations. We clearly see there that, in spite of the large differences in the energy gaps between the levels (e.g., see the data in Figure 3), the efficiency of the collisional excitations does not change much over the range of temperatures 


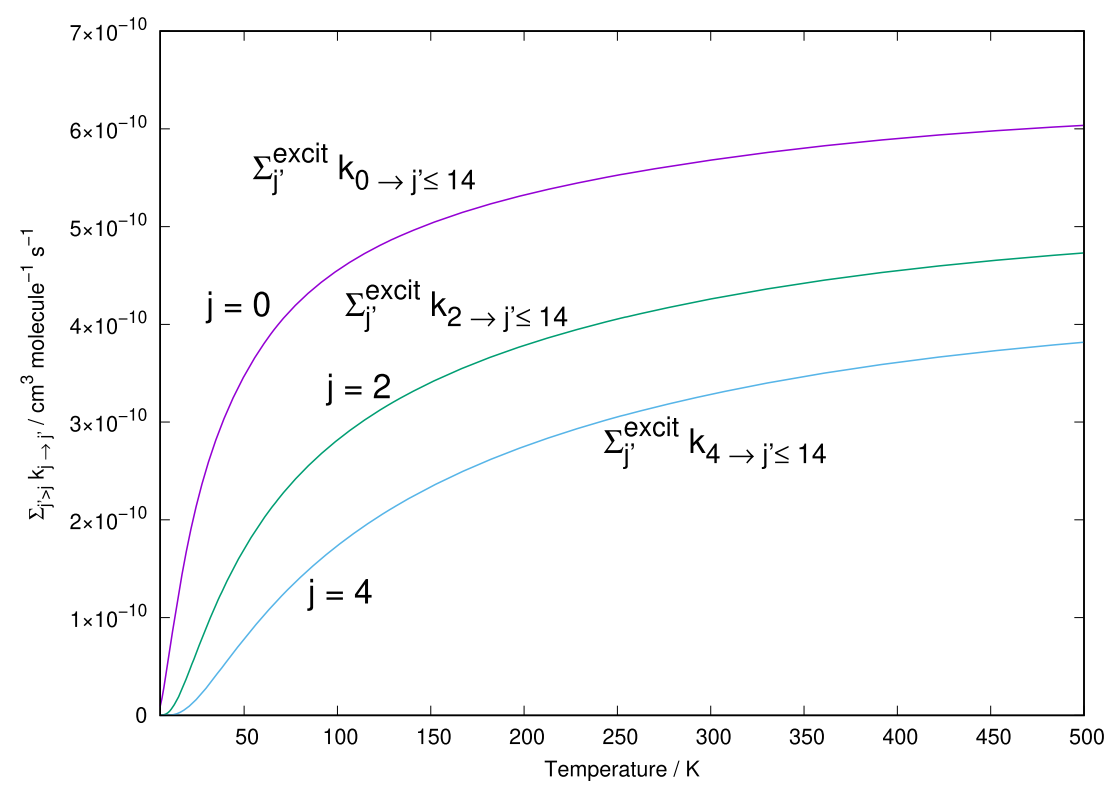

Figure 11. Computed excitation rate coefficients summed over all accessible final levels and starting from the lowest three rotational states of the present cation. Notice the enlarged scale of the ordinates.

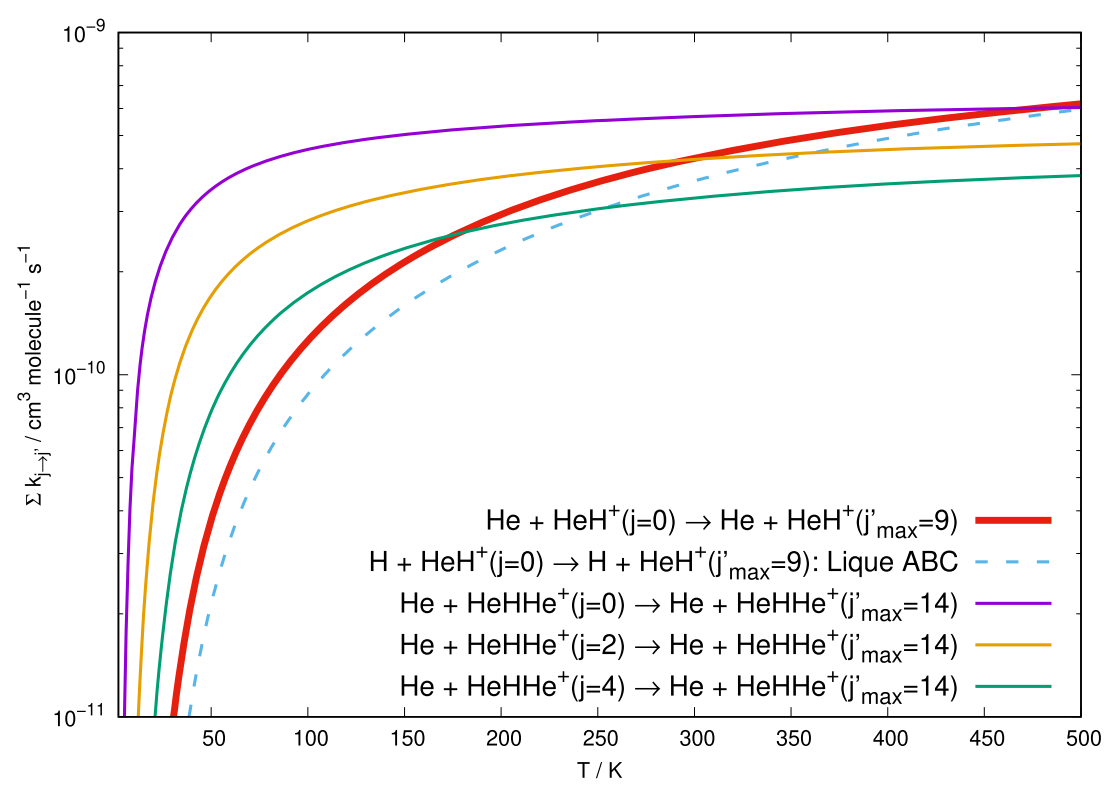

Figure 12. Comparison of the behavior of the summed excitation rates for the $\mathrm{HeHHe}^{+}$and the $\mathrm{HeH}^{+}$systems in collision with $\mathrm{He}$ and $\mathrm{H}$ atoms. The dashed thin line reports the calculations from ref 43 for collisions of $\mathrm{H}$ atoms with $\mathrm{HeH}^{+}$, while the thick solid line reports our earlier calculations from ref 40 on the $\mathrm{HeH}^{+}$collisions with $\mathrm{He}$ atoms. The present results for the $\mathrm{HeHH}^{+}$in collision with $\mathrm{He}$ atoms are given by the three thin solid lines of different colors.

examined. The range in size differences between the lowest excitation rate coefficient (that for the $j=0$ to $j=2$ process) to the one involving $\Delta j=4$ transitions between the most highly excited levels considered in this study (the $j=6$ to $j=10$ case) is on average a factor of 2 at most. It is therefore the strong anisotropic potential coupling discussed earlier, and the similar strength of the two anisotropic terms for $\lambda=2$ and $\lambda=4$ that dominates the outcomes of the inelastic dynamics, hence limiting the effects from the energy gaps in the excitation processes.

The data shown in Figure 10 present a different way of assessing the rotational excitation efficiency of the present system. We show results for three different temperature values, while each panel in turn reporting excitation rate coefficients for multiple transitions originating from a different level in each panel. The following considerations can be made:

(i) although we are considering transitions from $\Delta j=2$ to $\Delta j=10$, we see that the rate constant values change rather little along the four panels, especially when the higher temperatures are considered;

(ii) only at the lowest temperature a significant drop in rate constant value occurs as $\Delta j$ increases: we have seen from the previous figures reporting the individual rates that the $T$ dependence at the lower temperatures varies markedly as $\Delta j$ changes, hence the present effect;

(iii) the slow dependence of the inelastic rate coefficients on temperatures at higher $T$ values suggest that the 
efficiency of the energy transfer processes remains large over a broad range of environmental situations, hence underlying the good energy-storing efficiency expected from the present molecule under early universe conditions.

An additional set of indicators is shown by the data in Figure 11. The excitation rate coefficients are now summed over all of the accessible final states as the temperature values span the range discussed in this work. This quantity therefore provides a sort of global indicator of the excitation efficiency shown by the $\mathrm{HeHHe}^{+}$in collision with neutral He. Three different situations are shown in the figure, with different initial rotational states of the target being selected: $j=0, j=2$, and $j=4$, with the inclusion of all available excitation transitions from each of these levels.

All of the summed rate coefficients turn out to be fairly close in value, with the processes initiated with the cation in its ground-state rotational level being the largest, as should be expected from the dominance of the $j=0$ to $j=2$ transition seen in the previous sections. We further notice that the changes in size from the smallest to the largest quantity presented in Figure 11 are rather small, once more suggesting the overall large efficiency of the present excitation processes at the temperatures of interest.

As we have mentioned in the Introduction section, the possible presence of $\mathrm{HeHHe}^{+}$is also linked to the presence of the recently observed $\mathrm{HeH}^{+}$polar cation. Some of the paths to its formation and to the further formation of other $(\mathrm{He}, \mathrm{H})$ containing molecules have been recently discussed in ref 44 . We have also outlined some further possibilities in the Introduction section and will discuss them more specifically in future work. Since neutral $\mathrm{He}$ and neutral $\mathrm{H}$ atoms can interact with either of the above ionic molecules, we thought it to be of interest to compare the efficiency of the energy transfer processes involving rotational states of both of them when colliding with surrounding hydrogen atoms, especially since data already exist for the case of $\mathrm{HeH}^{+}$in collision with $\mathrm{H}$ from the earlier work reported in refs 43 and 40 .

We present a comparison in Figure 12, where different cases for the computed global, inelastic rate coefficients for both $\mathrm{HeH}^{+}$and $\mathrm{HeHHe}^{+}$in collision with $\mathrm{He}$ atoms are shown. Earlier results for the case of $\mathrm{HeH}^{+}$colliding with $\mathrm{H}$ atoms are also reported. ${ }^{43}$

The following comments can be made from a perusal of the data:

(i) In the lower range of temperatures, up to about 250$300 \mathrm{~K}$, the efficiency of the rotational energy transfer dynamics for the case of the present molecular ion $\mathrm{HeHHe}^{+}$, when in one of its lowest rotational states $j=0$ or $j=2$, is much larger than that found for $\mathrm{HeH}^{+}$. These states are also the most probable initial states at the expected temperature conditions in the ISM;

(ii) The collisional inelastic rate constants involving either $\mathrm{H}$ or $\mathrm{He}$ atoms interacting with $\mathrm{HeH}^{+}$are becoming of the same size as those for $\mathrm{HeHHe}^{+}$only at the highest temperatures considered in the present study, while remaining smaller at the lower temperatures.

The above results indicate that the present cation, if in existence where $\mathrm{HeH}^{+}$has been already found to be present, ${ }^{12}$ would be a more efficient participant to the energy-storing mechanisms induced by collisions with abundant species, like $\mathrm{He}$ and $\mathrm{H}$, present in the same environment. This is an interesting conclusion, suggesting that including such species within enlarged evolutionary models of the chemistry in the early universe would be a useful improvement (e.g., see ref 13).

From the variety of estimated baryon densities in the early universe discussed earlier, in fact, we already know that the baryon density $n_{\mathrm{b}}$ is proportional to the redshift value $z$ via the relationship: $(1+z)^{3}$ (see ref 21$)$. Hence, we can say that for values of $z$ varying between 200 and 5000, the corresponding $n_{\mathrm{b}}$ values would be varying between about $10^{-1} \mathrm{~cm}^{-3}$ and about $10^{3} \mathrm{~cm}^{-3}$. Such values would therefore bracket the likely range of densities for the $\mathrm{He}$ partner as one of the most abundant species that can be expected to exist at the recombination era of the early universe conditions, as discussed in ref 21 . To better understand the variations that such density values would have on the collisional astronomical times of the present species with the He atoms, it is convenient to introduce another useful, global indicator by defining a quantity, called $\tau$, obtained from the summed collisional relaxation rate coefficients multiplied by the available density estimates of the environment under consideration

$$
\tau(\mathrm{s})=\left[\left(\sum k_{j \rightarrow j^{\prime}}(T)\right) \cdot\left(n_{\mathrm{b}}\right)\right]^{-1}
$$

This quantity, given in units of s, can be obtained using the two extreme values of $n_{\mathrm{b}}$ mentioned earlier and summing over all our calculated rate coefficients $k_{j \rightarrow j^{\prime}}(T)$ discussed in the previous section. We therefore find that the purely collisional relaxation times provided by $\tau$, depending on the estimated baryon density range in the early universe models, can vary rather dramatically over more than 4 orders of magnitude from $10^{10} \mathrm{~s}$ at the lowest redshift values $(z=200)$ to about $10^{6} \mathrm{~s}$ at the higher redshift values of 5000. This result further underscores in more quantitative terms the role of collisiondriven energy exchanges in relation to the actual density conditions in which the molecular partners are operating.

\section{CONCLUSIONS}

One of the aims of the present study has been to consider the energy dissipation role, through rotational energy transfer paths, of a possible new entry among the early universe molecules, the $\mathrm{HeHHe}^{+}$cation, interacting with $\mathrm{He}$ atoms under the expected conditions suggested by the existing modeling studies (e.g., see ref 21). Although this cation has not yet been observed, its likely formation routes under the astronomical conditions have been studied in the recent literature (e.g., see refs 23,24 ) where various possibilities for its formation and energy dissipation paths were surmised.

We have pursued this specific aspect through direct quantum dynamics calculations and have obtained the potential energy surface for the interaction between this cation, treated in a first instance as being at its equilibrium bond distances (a rigid rotor target), and the $\mathrm{He}$ neutral atom using high-quality $\mathrm{ab}$ initio treatment. We have then employed it to generate the quantum inelastic cross sections for rotational excitation processes over a broad range of rotational states and further evaluated the corresponding inelastic rate coefficients up to about $500 \mathrm{~K}$ for the interstellar conditions. The results obtained have been illustrated in detail in the previous sections and indeed suggest that the strong anisotropy of the interaction potential is responsible for inducing rather large inelastic, rotational state-changing cross sections right from near-threshold collision energies, thereby generating the corresponding inelastic rate constants as being unexpectedly 
large for this cation and under the examined temperature conditions.

We have also shown various dynamical indicators and further compared the present findings with earlier results involving both $\mathrm{He}$ and $\mathrm{H}$ as collision partners of a polar cation present after the recombination era: the $\mathrm{HeH}^{+}$. This comparison indicates that $\mathrm{HeHHe}^{+}$yields larger inelastic rate coefficients under similar conditions as those where the $\mathrm{HeH}^{+}$ was studied, thus surmising that the inclusion of this molecule into the chemical networks modeling the kinetic evolution of the early universe would provide a more realistic description of the actual energy dissipation processes, which could occur under those conditions.

Although the present molecular cation has no permanent dipole moment at its equilibrium geometry, the existence of an asymmetric stretching mode with a substantial transition dipole as discussed in refs 23,24 could provide another collisional route to energy transfer dynamics. This possible option will be further discussed in future work in preparation in our laboratory.

\section{ASSOCIATED CONTENT}

\section{(s) Supporting Information}

The Supporting Information is available free of charge at https://pubs.acs.org/doi/10.1021/acs.jpca.1c01820.

Multipolar coefficients for the Legendre expansion of the new rigid rotor $\mathrm{PES}$ for the $\mathrm{HeHHe}^{+}$interaction with $\mathrm{He}$ are provided via a Fortran program routine and data files providing the computed inelastic rate coefficients for the rotational excitation of the title molecule in collision with $\mathrm{He}$ atoms (ZIP)

\section{AUTHOR INFORMATION}

\section{Corresponding Author}

F. A. Gianturco - Institut fur Ionen Physik und Angewandte Physik, Leopold-Franzens-Universitat, 6020 Innsbruck, Austria; (1) orcid.org/0000-0003-3962-530X; Email: francesco.gianturco@uibk.ac.at

\section{Authors}

L. González-Sánchez - Departamento de Química Física, University of Salamanca, 37008 Salamanca, Spain

E. Yurtsever - Department of Chemistry, Koc University, 34450 Istanbul, Turkey; (1) orcid.org/0000-0001-92459596

R. Wester - Institut fur Ionen Physik und Angewandte Physik, Leopold-Franzens-Universitat, 6020 Innsbruck, Austria; () orcid.org/0000-0001-7935-6066

Complete contact information is available at:

https://pubs.acs.org/10.1021/acs.jpca.1c01820

\section{Notes}

The authors declare no competing financial interest.

\section{ACKNOWLEDGMENTS}

F.A.G. and R.W. acknowledge the support by the Austrian Science Fund (FWF), Project No. 29558-N36. Funding by the Spanish Ministry of Science and Innovation (grant PGC2018096444-B-I00) is also acknowledged by L.G.-S.

\section{REFERENCES}

(1) Baccarelli, I.; Gianturco, F. A.; Schneider, F. Stability and Fragmentation of Protonated Helium Dimers from ab Initio Calculations of Their Potential Energy Surfaces. J. Phys. Chem. A 1997, 101, 6054.

(2) Filippone, F.; Gianturco, F. A. Charged Chromophoric Units in Protonated Rare-gas Clusters: A Dynamical Simulation. Europhys. Lett. 1998, 44, 585.

(3) Bovino, S.; Tacconi, M.; Gianturco, F. A.; Galli, D. Ion Chemistry in the Early Universe Revisiting the Role of $\mathrm{HeH}^{+}$with New Quantum Calculations. Astron. Astrophys. 2011, 529, A140.

(4) Lepp, S.; Stancil, P.; Dalgarno, A. The chemistry of the Early Universe. J. Phys. B: At., Mol. Opt. Phys. 2002, 35, R57-R80.

(5) Hogness, T.; Lunn, E. The ionization of Hydrogen by electron impact as interpreted by positive ray analysis. Phys. Rev. 1925, 26, 4455.

(6) Tolliver, D.; Kyrala, G.; Wing, W. Observation of the Infrared Spectrum of helium-hydride molecular ion ${ }^{4} \mathrm{HeH}^{+}$. Phys. Rev. Lett. 1979, 43, 1719-1722.

(7) Bernath, P.; Amano, T. Detection of the infrared fundamental band of $\mathrm{HeH}^{+}$. Phys. Rev. Lett. 1982, 48, 20-22.

(8) Crofton, M.; Altman, R.; Haese, N.; Oka, T. Infrared spectra of ${ }^{4} \mathrm{HeH}^{+},{ }^{4} \mathrm{HeD}^{+},{ }^{3} \mathrm{HeH}^{+}$, and ${ }^{3} \mathrm{HeD}^{+}$. J. Chem. Phys. 1989, 91, 58825886.

(9) Perry, A. J.; Hodges, J.; Markus, C.; Kocheril, G.; McCall, B. High-precision sub-Doppler infrared spectroscopy of the $\mathrm{HeH}^{+}$ion. $J$. Chem. Phys. 2014, 141, No. 101101.

(10) Matsushima, F.; Oka, K.; Takagi, T. Observation of the rotational spectra of ${ }^{4} \mathrm{HeH}^{+},{ }^{4} \mathrm{HeD}^{+},{ }^{3} \mathrm{HeH}^{+}$and ${ }^{3} \mathrm{HeD}^{+}$. Phys. Rev. Lett. 1997, 78, 1664-1666.

(11) Liu, D.; Ho, W.; Oka, T. Infrared spectroscopy and equilibrium structure of $\mathrm{H}_{2} \mathrm{O}^{+}$. J. Chem. Phys. 1987, 87, 2442.

(12) Güsten, R.; Wiesemeyer, H.; Neufeld, D.; Menten, K. M.; Graf, U. U.; Jacobs, K.; Klein, B.; Ricken, O.; Risacher, C.; Stutzki, J.; et al. Astrophysical detection of the helium hydride ion $\mathrm{HeH}$. Nature 2019, $568,357$.

(13) Bovino, S.; Galli, D. First Molecule Still Animates Astronomers. Science 2019, 365, 639.

(14) Kojima, T.; Kobayashi, N.; Kaneko, Y. Cationic complexes of hydrogen with He. Z. Phys. D: At., Mol. Clusters 1992, 23, 181-185.

(15) Lundberg, L.; Bartl, P.; Leidlmair, C.; Scheier, P.; Gatchell, M. Protonated and cationic Helium clusters. Molecules 2020, 25, 1066.

(16) Balta, B.; Gianturco, F. Structural properties and quantum effects in protonated helium clusters. Chem. Phys. 2000, 254, 203213

(17) Stephan, C.; Fortenberry, R. The interstellar formation and spectra of the nobel gas, proton-bound $\mathrm{HeHHe}^{+}$and $\mathrm{HeHAr}^{+}$ complexes. Mon. Not. R. Astron. Soc. 2017, 469, 339-346.

(18) Grandinetti, F. Helium chemistry: A survey of the role of the ionic species. Front. Chem. 2020, 8, 462.

(19) Shiell, R.; Hu, X. K.; Hu, Q. C. J.; Hepburn, J. W. Threshold Ion-pair Production spectroscopy (TIPPS) of $\mathrm{H}_{2}$ and $\mathrm{D}_{2}$. Faraday Discuss. 2000, 115, 331.

(20) Bovino, S.; Tacconi, M.; Gianturco, F. A.; Galli, D. Ion Chemistry in the Early Universe Revisiting the Role of $\mathrm{HeH}^{+}$with New Quantum Calculations. Astron. Astrophys. 2011, 529, A140.

(21) Galli, D.; Palla, F. The Dawn of Chemistry. Annu. Rev. Astron. Astrophys. 2013, 51, 163.

(22) Meijerink, R.; Spaans, M.; Israel, F. Diagnostics of irradiated dense gas in galaxy nuclei. II. A grid of XDR and PDR models. Astron. Astrophys. 2007, 461, 793-811.

(23) Fortenberry, R. C.; Wiesenfeld, L. A Molecular Candle where few molecules shine: $\mathrm{HeHHe}^{+}$. Molecules 2020, 25, 42183.

(24) Panda, A. N.; Sathyamurthy, N. Bound and Quasibound States of $\mathrm{He}_{2} \mathrm{H}^{+}$and $\mathrm{He}_{2} \mathrm{D}^{+}$. J. Phys. Chem. A 2003, 107, 7125 .

(25) Töpfer, M.; Jensen, A.; Nagamori, H.; Kohguchi, K.; Szidarovsky, T.; CsaİszaIIr, A.; Schlemmer, S.; Asvany, O. Spectroscopic signatures of $\mathrm{HHe}_{2}{ }^{+}$and $\mathrm{HHe}_{3}{ }^{+}$. Phys. Chem. Chem. Phys. 2020, 22, 22885-22888. 
(26) Adams, N.; Bohme, E.; Ferguson, D. K. Reactions of $\mathrm{He}_{2}{ }^{+}, \mathrm{Ne}_{2}{ }^{2}$ $+\mathrm{Ar}_{2}{ }^{+2}$ and Rare-Gas Hydride Ions with Hydrogen at 200K. J. Chem. Phys. 1970, 52, 5101-5110.

(27) Xu, W.; Zhang, P. Accurate Study on the Quantum Dynamics of the $\mathrm{He}+\mathrm{HeH}^{+}\left(\mathrm{X}^{1} \Sigma^{+}\right)$Reaction on A New ab Initio Potential Energy Surface for the Lowest $1^{1} \mathrm{~A}^{\prime}$ Electronic Singlet State. J. Phys. Chem. A 2013, 117, 1406.

(28) Ziurys, L. M. The chemistry in circumstellar envelopes of evolved stars: Following the origin of the elements to the origin of life. Proc. Natl. Acad. Sci. U.S.A. 2006, 103, 12274.

(29) Werner, H. J.; Knowles, P. J.; Knizia, G.; Manby, F. R.; Schutz, $\mathrm{M}$. Molpro: a general-purpose quantum chemistry program package. Wiley Interdiscip. Rev.: Comput. Mol. Sci. 2012, 2, 242-253.

(30) Werner, H.; Knowles, P.; Manby, F.; Black, J.; Doll, K.; Hesselmann, A.; Kats, D.; Koehn, A.; Korona, T.; Kreplin, D. et al. MOLPRO, Version 2019.2, A Package of Ab Initio Programs, 2019. https://www.molpro.net.

(31) Taylor, J. R. Scattering Theory: The Quantum Theory of Nonrelativistic Collisions; Dover, 2006.

(32) Arthurs, A. M.; Dalgarno, A. The theory of scattering by a rigid rotator. Proc. R. Soc. London, Ser. A 1960, 256, 540.

(33) Secrest, D. Rotational Excitation-I: The Quantal Treatment. In Atom - Molecule Collision Theory; Bernstein, R. B., Ed.; Plenum: New York, 1979.

(34) Kouri, D.; Hoffman, D. A Tutorial on Computational Approaches to Quantum Scattering. In Multiparticle Quantum Scattering With Applications to Nuclear, Atomic and Molecular Physics; Truhlar, D. G.; Simon, B., Eds.; Springer: New York, NY, 1997; Vol. 89.

(35) Hutson, J. Coupled channel methods for solving the boundstate Schroedinger equation. Comput. Phys. Commun. 1994, 84, 1-18.

(36) Gianturco, F. The Transfer of Molecular Energies by Collisions: Recent Quantum Treatments; Lecture Notes in Chemistry; Springer Verlag: Berlin, 1979.

(37) Martinazzo, R.; Bodo, E.; Gianturco, F. A. A modified VariablePhase algorithm for multichannel scattering with long-range potentials. Comput. Phys. Commun. 2003, 151, 187.

(38) López-Durán, D.; Bodo, E.; Gianturco, F. A. ASPIN: An all spin scattering code for atom-molecule rovibrationally inelastic cross sections. Comput. Phys. Commun. 2008, 179, 821.

(39) González-Sánchez, L.; Gianturco, F. A.; Carelli, F.; Wester, R. Computing rotational energy transfers of $\mathrm{OD}^{-} / \mathrm{OH}^{-}$in collisions with $\mathrm{Rb}$ : isotopic effects and inelastic rates at cold ion-trap conditions. New J. Phys. 2015, 17, No. 123003.

(40) Gianturco, F. A.; Giri, K.; González-Sánchez, L.; Yurtsever, E.; Sathyamurthy, N.; Wesre, R. Energy-transfer quantum dynamics of $\mathrm{HeH}^{+}$with $\mathrm{He}$ atoms: Rotationally inelastic cross sections and rate coefficients. J. Chem. Phys. 2021, 154, No. 054311.

(41) Massey, H. Collisions between atoms and molecules at ordinary temperatures. Rep. Prog. Phys. 1949, 12, 248.

(42) Hernández Vera, M.; Gianturco, F. A.; Wester, R.; da Silva, H., Jr.; Dulieu, O.; Schiller, S. Rotationally inelastic collisions of $\mathrm{H}_{2}{ }^{+}$ions with He buffer gas: Computing cross sections and rates. J. Chem. Phys. 2017, 146, No. 124310.

(43) Desrousseaux, B.; Lique, F. Collisional Energy Transfer in the $\mathrm{HeH}^{+}-\mathrm{H}$ reactive System. J. Chem. Phys. 2020, 152, No. 074303.

(44) Zicler, E.; Parisel, O.; Pauzat, F.; Ellinger, Y.; BacchusMontabonel, M.-C.; Maillard, J.-P. Search for hydrogen-helium molecular species in space. Astron. Astrophys. 2017, 607, A61. 